\title{
Lusioersily
}

\section{A Survey of Radio Propagation Channel Modelling for Low Altitude Flying Base Stations}

Ahmad, A., Cheema, A. A., \& Finlay, D. (2020). A Survey of Radio Propagation Channel Modelling for Low Altitude Flying Base Stations. Computer Networks, 171, [107122]. https://doi.org/10.1016/j.comnet.2020.107122

Link to publication record in Ulster University Research Portal

\author{
Published in: \\ Computer Networks
}

Publication Status:

Published (in print/issue): 22/04/2020

DOI:

10.1016/j.comnet.2020.107122

\section{Document Version}

Author Accepted version

\section{General rights}

Copyright for the publications made accessible via Ulster University's Research Portal is retained by the author(s) and / or other copyright owners and it is a condition of accessing these publications that users recognise and abide by the legal requirements associated with these rights.

\section{Take down policy}

The Research Portal is Ulster University's institutional repository that provides access to Ulster's research outputs. Every effort has been made to ensure that content in the Research Portal does not infringe any person's rights, or applicable UK laws. If you discover content in the Research Portal that you believe breaches copyright or violates any law, please contact pure-support@ulster.ac.uk. 


\title{
A Survey of Radio Propagation Channel Modelling for Low Altitude Flying Base Stations
}

\author{
Abrar Ahmad, Adnan Ahmad Cheema*, Dewar Finlay \\ School of Engineering, Ulster University, Jordanstown, BT37 0QB, UK
}

\begin{tabular}{l} 
A R T I C L E I N F O \\
\hline Article history: \\
Received 00 August 19 \\
Received in revised form 00 Month 00 \\
Accepted 00 Month 00 \\
\hline Keywords: \\
Air-to-Ground, Unmanned Aerial \\
Vehicles (UAVs), Low Altitude \\
Platform (LAP), Radio Propagation \\
Channel (RPC), Flying Base Station, \\
Disaster Management
\end{tabular}

\section{Introduction}

The use of unmanned aerial vehicles (UAVs) or commonly known as drones, is progressing tremendously in our everyday life. In UK and US, small UAVs with weight less than $20 \mathrm{~kg}$ in permitted areas (e.g. a specific distance away from aerodrome boundary and flying without being into conflict with people or properties) can be flown on an altitude less than 400 feet (i.e. $122 \mathrm{~m}$ ) without any license [1,2]. Here, the altitude is considered from the surface of the earth whether the operational area belongs to hilly, undulating or flat surface. Now a days, UAVs are in use in various applications like transportation of goods or first aid, inspection of crops in farming, surveillance by government agencies, filming movies, live coverage of concerts and sports, remote sensing, search and rescue and many more on account of their small size, cost-effective, agility, nonpiloted and low altitude flying ability [3]. According to recent research [4], the market value of UAVs will tend to grow up to $\$ 12.6$ billion by 2025 .

Apart from aforementioned applications of small UAVs, they were proposed to assist in providing improved wireless network coverage by manoeuvring as low altitude platforms (LAPs: from tens to few hundreds of meter) [5-12]. They can be deployed as flying base stations (BSs) or relays to improve wireless network coverage [13]. Particularly, in the scenarios demanding high data rate on emergency needs and in the areas where signals get severely deteriorated due to various obstacles [14], [15].

In case of natural disasters, already existing terrestrial communication infrastructures are prone to get severely damaged (e.g. Indonesia tsunami (2004), Gulf Coast Katrina hurricane (2005), Haiti Earthquake (2010) [16] and Japan Earthquake (2011) [17]). It is noticed that the number of natural disasters tends to increase in every decade [18]. Generally, the major issue faced with severely damaged terrestrial communication infrastructures in the result of large scale disasters is that the enduring BSs get congested and due to this quality of service gets compromised [19]. The first 72 hours after a disaster are of vital importance for the first responders to accomplish effective search and rescue missions [14]. Therefore, such unexpected scenarios demand the provision of wireless network coverage on an emergency basis for strategical disasters management [20]. Authors in [21], analysed the performance of several algorithms to be used in UAV assisted networks for visual based searching of a victim with time to find the victim as an optimization parameter.

The existing terrestrial communication infrastructure support systems (e.g. deployment of the 
cell on wheels (COW) and cell on light trunks (COLT)) has several shortcomings to meet the need of wireless network coverage for disaster management [22]. For example, time taken to physically arrive in the affected areas and network congestion are the basic shortcomings in COW and COLT. Furthermore, fifthgeneration $(5 \mathrm{G})$ and beyond $5 \mathrm{G}(\mathrm{B} 5 \mathrm{G})$ are expected to have improved resilience in wireless network coverage in case of emergencies or unavailability of existing terrestrial communication infrastructure [23]. How non-terrestrial networks (e.g. drone assisted) will assist to improve resilience in future wireless networks coverage, is an important dimension to explore.

One possible solution might be satellite-based communication systems however, they have their own limitations [24]. For example, the geostationary satellites have a large distance from the surface of the earth and face large delays. Whereas the nongeostationary satellites are complex, costly to launch, and they can only be launched in the limited number of orbits due to which available communication links are expensive. Another possible solution could be high altitude platforms (HAPs: on an altitude of $20-50 \mathrm{~km}$ ) $[25,26]$, however, they have own limitations $[27,28]$. For example, cost, hardware complexity, time taking deployment and configuration, and limited data rate are the major issues. HAPs may be useful when the wireless network coverage needs to be provided on a very large coverage area for longer endurance. Facebook and Google are currently working on a project for HAP to provide internet access [29]. However, in the scenarios of emergencies for being quickly deployable, HAPs are not an adequate solution to support terrestrial communication infrastructures. On the other hand, LAPs do not have such kind of issues. For example, they can be ready to deploy, easily reconfigurable, adaptive altitude, costeffectiveness, and more chances of having short distance line-of-sight (LOS) communication links with the receiver for providing high capacity and low latency $[9,30]$. UAVs enabled flying BSs and relays are considered to assist terrestrial communication infrastructure for improved wireless network coverage [31-35]. GSMA (Groupe Spéciale Mobile Association) encouraged the use of UAVs in disaster management for surveillance to assist the first responders and flying BSs or relays to make the partially damaged terrestrial mobile networks functional [36]. The integration of UAVs with existing terrestrial communication infrastructures can enhance capacity and coverage with energy efficiency and reliability in future wireless networks, particularly for the scenarios of emergencies or hard to reach areas for broadcasted signals [12]. In [15], researchers highlighted how UAVs connected with terrestrial BS can assist to provide wide area coverage, secure identification and authorization, and interoperability among globally evolving wireless network coverage. Furthermore, the communication link from flying BS to a receiver can have another advantage of controlled mobility of UAV. For example, in the need of high data rate, if LOS link is established with the receiver, the motion of UAV can be switched to the only hover for maintaining the LOS link for improved communication between flying BS and the receiver.

One of the major challenges in designing UAVs enabled wireless network coverage is the modelling of the radio propagation channel (RPC) $[9,12,27,37]$. The better understanding of the RPC will be helpful to model the fading (large scale and small scale) effects caused by the environment and design the reliable wireless communication systems. The radio propagation in flying BSs will significantly differ from existing terrestrial communication infrastructures. The basic constraints behind these differences consist of communication link distance variation, ground reflections, multipath fading effects, antenna orientation, interference and jamming, the effect of electronics equipment of UAV and vibrations of the UAV [8]. Fig. 1 shows the possible effects (to count few) on the signal propagation from a UAV to a receiver.

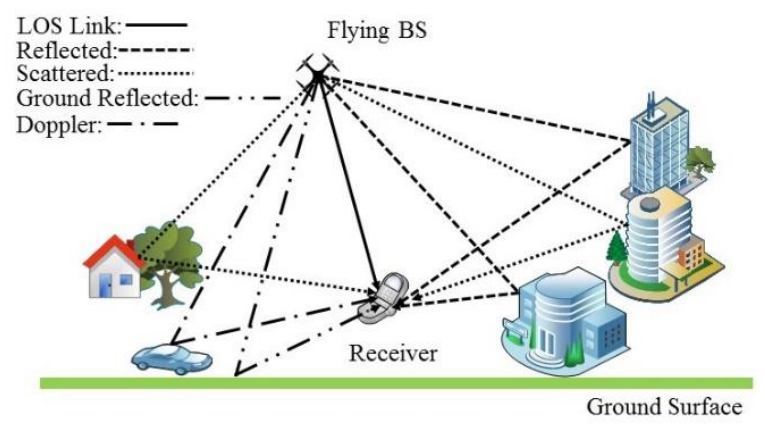

Fig. 1. An illustration of signal propagation from a UAV to the receiver on the ground

The $5 \mathrm{G}$ is expected to be launched in various parts of the world in 2020 and its spectrums will occupy majorly in three bands: low band (below $1 \mathrm{GHz}$ ), mid band $(1-6 \mathrm{GHz})$, and high band $(6-100 \mathrm{GHz})$ [38]. Mid band and high band in most parts of Europe (including the UK) will be auctioned around frequencies of $3.5 \mathrm{GHz}$ and $26 \mathrm{GHz}$ respectively [38]. The mid band around $3.5 \mathrm{GHz}$ could be useful in search and rescue operations on account of being able to penetrate into a vast variety of materials [39] (e.g. walls, doors, building, and beneath the ground to get images of buried objects) along with required wireless communication services. Therefore, modelling of the RPC in the mid band can contribute significantly in the research of UAVs enabled assistance to the terrestrial 
communication infrastructure for its improved wireless network coverage. On the other hand, it is also important to model the RPC in spectrums already being used in the existing cellular networks (from 800 $\mathrm{MHz}$ to $2600 \mathrm{MHz}$ ) [40].

$3^{\text {rd }}$ Generation Partnership Project (3GPP) started focusing to handle required data rate, latency, altitude and speed limitations, interference mitigation, evaluation scenarios and channel modelling in low altitude UAV based communication systems [41,42]. Furthermore, International Telecommunication Union (ITU) emphasized the use of UAVs as a relay for transmitting wireless sensor networks (WSNs) information from affected areas to computer servers for assistance in disaster management [43].

This paper provides a detailed survey (section 2 ) of the RPC quantification and modelling that includes both measurement based and simulation based investigations for UAV enabled future wireless networks. Section 0 highlight open research problems. The future research directions are provided in section 4 along with proposed use cases which are expected to be important for UAV enabled networks and required further investigation for radio propagation channel modelling. Finally, the paper is concluded in section 5.

\section{Literature Review}

This section provides a comprehensive literature review of the RPC modelling for UAV enabled wireless networks along with the considered use cases and the limitations. Several survey papers have been published in the literature on the research of UAVs enabled wireless network coverage to summarize the use cases, challenges, resources management, and future perspectives $[8,12,16,34,44-51]$. In this review paper, our focus is to summarize the on-going research work relating to the radio propagation channel modelling for low altitude UAV based wireless networks. This review will summarize the platforms (i.e. hardware and software) and relating parameters for channel modelling, modelling approaches (i.e. measurements or simulation), scenarios, key findings and limitations. These limitations are key to define the proposed use cases and relating research gaps, as discussed in section 4, to improve network coverage for disaster management and upcoming market of $5 \mathrm{G}$ and $\mathrm{B} 5 \mathrm{G}$.

This section is explicitly divided into two subsections based on the type of modelling approach: 1) measurement and 2) simulation based channel modelling. In order to remain consistent, few terminologies need to be defined first. The downlink from a UAV to a receiver and terrestrial $\mathrm{BS}$ are respectively referred to as air-to-ground $(\mathrm{A} 2 \mathrm{G})$ and air-to-BS (A2B), as shown in Fig. 2. While the uplink from the receiver and terrestrial $\mathrm{BS}$ to $\mathrm{UAV}$ are referred to as ground-to-air (G2A) and BS-to-air (B2A), respectively. The distance from a UAV to the receiver and ground level refers as link-distance and altitude and the acute angle between link-distance and horizontal distance is refer to an elevation angle. The distance from ground level to the receiver is referred to as receiver height. Fig. 3 shows the graphical representation of these distances and the elevation angle.

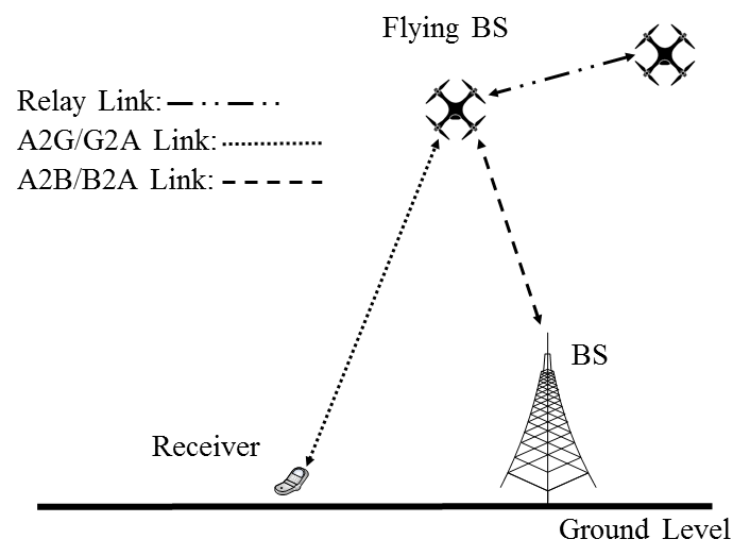

Fig. 2. Types of channel links in the UAV based wireless network

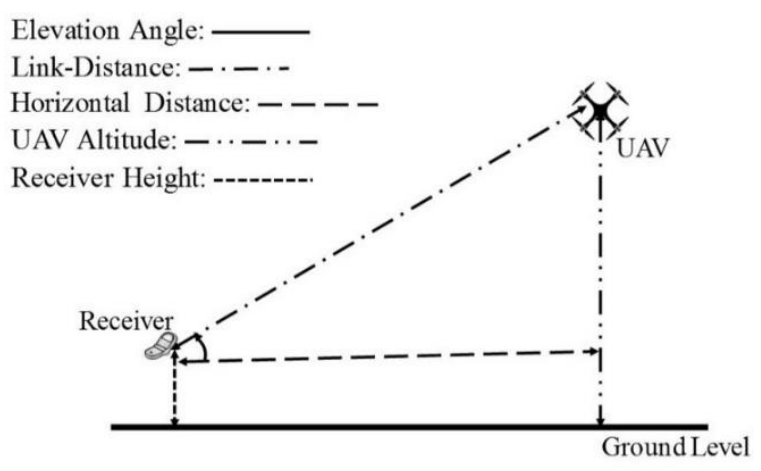

Fig. 3. Types of distances and elevation angle in UAV based wireless network

\subsection{Measurement Based Channel Modelling}

Radio waves when propagate undergo several types of losses and environmental effects (e.g. large scale and small scale fading) depending upon the type of environment, distance travelled and transmitted frequency [52]. Mainly, two methods were used to investigate the RPC modelling by measuring: (1) channel impulse response (CIR) by an appropriate channel sounding equipment [52-56] and using CIRs to compute both large scale fading (e.g. path loss and shadowing) and/or small scale fading parameters (e.g. delay spread) and (2) received power, which can only 
provide large scale fading parameters. Each measurement based attempt in the literature to model the RPC is classified into one of the four categories: A2G, G2A, A2B and B2A, and will be discussed in following sub-sections.

\subsubsection{A2G Channel Modelling}

Received power and throughput were measured in [57] for the open area on altitude $20-120 \mathrm{~m}$ in the frequency spectrum of $2.4 \mathrm{GHz}$ and large scale fading analysis was provided. Measurement campaign with CIR based large and small scale fading analysis however, limited to an open area and very low altitude (16 m) was performed in [58]. In [59], only path loss and throughput were measured in open area scenarios within cellular (900 MHz and $1800 \mathrm{MHz}$ ) and $\mathrm{Wi}-\mathrm{Fi}$ ( $5 \mathrm{GHz}$ ) bands for a maximum altitude of $30 \mathrm{~m}$. In the continuation of this research [60], while UAV was hovering and flying in a circular path with $6 \mathrm{~m} / \mathrm{s}$ speed, bit error rate (BER) and throughput were measured. A measurement campaign in the open area was done for large scale and small scale A2G channel modelling in frequency bands around $1.8 \mathrm{GHz}$ and 5.7 $\mathrm{GHz}$ for LOS communication link with an altitude of $30 \mathrm{~m}$ [61]. However, the RPC modelling needs to be further investigated for the partially and fully obstructed channel because channel models in LOS distinctly differ from that of non-LOS (NLOS) [62]. An A2G channel modelling in the open area by investigating both large and small scale parameters within $3.4-3.8 \mathrm{GHz}$ frequencies was done in [63]. Another measurement campaign limited to open area and $40 \mathrm{~m}$ altitude in $1.2 \mathrm{GHz}$ band for $\mathrm{A} 2 \mathrm{G}$ channel modelling was done in [64]. The results showed less multipath propagation for higher altitudes. A measurement based effort was done for channel modelling within altitude ranges from $50-950 \mathrm{~m}$, horizontal distance up to $70 \mathrm{~km}$ and frequency bands around $785 \mathrm{MHz}$ and $2160 \mathrm{MHz}$ in LOS communication scenarios [65]. This measurement campaign for channel modelling was limited to only large scale fading and it was not a small UAV based, rather an aerial ship-based communication. The channel characteristics may differ when a small UAV is flown under altitude of $122 \mathrm{~m}$. In [66], UAV to vehicle LOS channel was analysed in terms of packet delivery ratio in the frequency band of $5 \mathrm{GHz}$ on two fix altitudes i.e. $40 \mathrm{~m}$ and $100 \mathrm{~m}$. Packet delivery ratio was observed greater for higher altitude.

\subsubsection{G2A Channel Modelling}

Comprehensive measurement campaigns [67-69] for large scale and small scale channel modelling from a tower to piloted aircraft up to $20 \mathrm{~km}$ altitude within frequency bands $0.968 \mathrm{GHz}$ and $5.06 \mathrm{GHz}$ were studied in open area, over the mountainous and surface of the sea. Significant variations in small scale fading parameters for larger link-distances were observed which possibly depicted the reflections from the water surface. Yet, G2A channel modelling for relatively low altitudes for smaller UAVs lacks and required further investigation.

\subsubsection{B2A Chanel modelling}

B2A channel was investigated in terms of measured received power and adjacent cell interference in $2 \mathrm{GHz}$

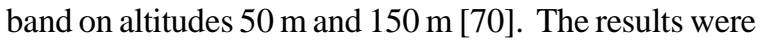
compared with the study of channel modelling from BS to a moving receiver (in a car) and B2A communication link was overall found to be better. In [71], a comprehensive measurement campaign in LOS scenarios was carried for B2A channel modelling on different altitudes and link-distances in $2.5 \mathrm{GHz}$ band. Overall, the results described that with larger altitudes and link-distances the fluctuations in the large and small scale parameters are significant. The work was a significant contribution for modelling of B2A channel, however, further adequate use cases for disaster management are needed to be studied e.g. including the effect of disaster debris on earth in various environments or weather conditions.

\subsubsection{A2B channel modelling}

The channel between a UAV and mobile network in the open area was modelled using large scale parameters and signal to interference and noise ratio (SINR) in $800 \mathrm{MHz}$ band [72].

In this sub-section 2.1, measurement based channel modelling attempts in different scenarios are useful as an initiative, however, more comprehensive investigations are further required particularly for relatively higher altitudes [42,73], in $5 \mathrm{G} \mathrm{mid/high}$ band in obstructed LOS and NLOS use cases and with mobility factors (e.g. either receiver is moving slowly or in a vehicle). In addition, channel modelling in the use cases with a flying UAV and continuously transmitting while ground receiver static/moving is important to be discussed. Authors in [74,75], modelled vehicle-to-vehicle channel for moving scatterers by considering Doppler effects in dynamic scenarios and such channel models can provide a base for dynamic scenarios (UAV, scatterers and/or receiver are in motion) in UAV enabled networks.

Table 1 summarizes the RPC channel models which have been used for measurement based channel modelling. The Log-distance path loss model has been widely used for scenarios relating LOS and open area. Apart from already used models as given in Table 1, further channel modelling approaches can be adopted for different scenarios [55]. 
Table 1. Summary of the RPC Models used in Surveyed papers

\begin{tabular}{|l|l|l|}
\hline \multicolumn{1}{|c|}{ Channel Model } & \multicolumn{1}{|c|}{ (Reference / Link Type) } & \multicolumn{1}{c|}{ Adopted Scenarios } \\
\hline $\begin{array}{l}\text { Log-distance Path Loss Model } \\
\text { (with/without modification) }\end{array}$ & $\begin{array}{l}\text { ([57] / A2G), ([58] / A2G), ([59] / A2G), } \\
([60] / \mathrm{A} 2 \mathrm{G}),([61] / \mathrm{A} 2 \mathrm{G}),([67-69] /\end{array}$ & Open area, LOS \\
\hline G2A), ([71] / B2A), ([72] / A2B) & $\begin{array}{l}\text { Airship (altitude from 50 - } \\
950 \text { m) communication with } \\
\text { a vehicle. }\end{array}$ \\
\hline $\begin{array}{l}\text { Modified COST - 2100 model } \\
\text { actual results) }\end{array}$ & $([65] / \mathrm{A} 2 \mathrm{G})$ & $\begin{array}{l}\text { LOS over water and in urban } \\
\text { environment. }\end{array}$ \\
\hline
\end{tabular}

\subsection{Simulation Based Channel Modelling}

Simulation based investigations of the RPC models were mostly done in the mid band and high band of $5 \mathrm{G}$ spectrum. The following section comprehensively describes the published literature related to RPC modelling for three channel links: A2G, G2A and B2A. To best of authors' knowledge, none of the publication was found relating the use cases for A2B.

\subsubsection{A2G Channel Modelling}

The probability of availability of LOS link and elevation angle dependent large scale fading were studied in $2-6 \mathrm{GHz}$ band for an altitude of $22 \mathrm{~km}$ [76]. In [77], the RPC from an aircraft to the receiver on the ground was modelled as a function of altitude and the horizontal distance. The model was based on a strong assumption that all multipath components (MPCs) were within the elliptical planar region. A ray-tracing simulation based A2G channel was modelled in hilly areas within frequencies from $200 \mathrm{MHz}$ to $5 \mathrm{GHz}$ in [78]. The presented results contained elevation angle dependent large scale and small fading analysis as well as probabilities of LOS, obstructed LOS and NLOS links. Another elevation angle dependent path loss for altitude up to $200 \mathrm{~m}$ was modelled in various LOS scenarios by using Wireless InSite Simulator [27]. In $2.4 \mathrm{GHz}$ band, only large scale fading was modelled based on elevation angle for altitudes $100-2000 \mathrm{~m}$ by using Wireless InSite Simulator in LOS and NLOS scenarios [79]. In [80], UAV assisted A2G channel in the cellular network was investigated in terms of probability of SINR greater than a certain threshold and dependence of UAV altitude and path loss exponent on the area spectral efficiency. Researchers in [81] and [82], modelled three-dimensional geometry-based (cylindrical and ellipsoidal respectively) A2G multipleinput multiple-output (MIMO) channels.

\subsubsection{G2A channel modelling}

In [83], a simulation based analysis of communication link for rescue vehicles (in terms of probability of received SNR greater than a threshold) against the UAV altitude up to $1000 \mathrm{~m}$ was performed for different transmit powers, number of vehicles and the coverage area.

\subsubsection{B2A Chanel modelling}

A simulation based study was carried out for unwanted interferences coming from adjacent BSs to UAV along with taking into account the coverage probability of a terrestrial BS and several UAV altitudes [84]. The result described that lowering the heights of terrestrial BSs, limiting the UAV altitude and down tilting the terrestrial BS antennas can be beneficial for optimized coverage towards both UAV and receivers.

In above all referred simulation based RPC investigations, the research attempts are mostly limited to LOS communication with several assumptions. Therefore, further simulation based campaigns are required for use cases in shopping malls, high-rise buildings and relating disasters. Furthermore, NYUSIM simulator can be useful to investigate simulations based RPC in various frequency bands [85].

The summary of channel modelling investigations including both measurement based and simulation based is provided in Table 2 and Table 3 . This summary includes parameters of investigations, scenarios, type of link and highlight their key findings. Following list of abbreviation is used in both Table 2 and Table 3.

$\begin{array}{ll}\text { Abbreviation } & \text { Description } \\ \text { COMM } & \text { Communication } \\ \text { Tx } & \text { Transmitter } \\ \text { Rx } & \text { Receiver } \\ \text { Freq } & \text { Frequency } \\ \text { DDP } & \text { Distance Dependent Path loss } \\ \text { PED } & \text { Path loss-Elevation angle-Dependent } \\ \text { PLE } & \text { Path Loss Exponent } \\ \text { PDP } & \text { Power Delay Profile } \\ \text { RMS-DS } & \text { Root Mean Square-Delay Spread } \\ \text { RKF } & \text { Rician-LOS K-Factor } \\ \text { DSSS-CCS } & \text { Direct Sequence Spread Spectrum } \\ & \text { Correlator Channel Sounder } \\ \text { RSS } & \text { Received Signal Strength } \\ \text { PDF } & \text { Probability Density Function }\end{array}$


Table 2. Summary of measurement based channel modelling and relating parameters

\begin{tabular}{|c|c|c|c|c|c|c|c|c|}
\hline Ref & $\begin{array}{l}\text { Freq } \\
\text { MHz } \\
\end{array}$ & Applications & Scenario & Type & $\begin{array}{c}\text { Main } \\
\text { Findings }\end{array}$ & $\begin{array}{c}\text { Studies } \\
\text { Parameters }\end{array}$ & $\begin{array}{c}\text { Distance }(\mathrm{m}) ; \\
\text { Tx height }(\mathrm{m}) \\
\text { Rx height }(\mathrm{m}) \\
\text { v }(\mathrm{m} / \mathrm{s})\end{array}$ & $\begin{array}{l}\text { Sounding } \\
\text { method } \\
\text { and/or } \\
\text { equipment }\end{array}$ \\
\hline [57] & 2400 & $\begin{array}{l}\text { Military, search, } \\
\text { tracking, } \\
\text { surveillance }\end{array}$ & $\begin{array}{l}\text { Open area, } \\
\text { campus } \\
\text { building in } \\
\text { LOS Com }\end{array}$ & A2G & $\begin{array}{l}\text { PLE }=2.6 \text { and } \\
2.5\end{array}$ & $\begin{array}{l}\text { RSS, } \\
\text { throughput }\end{array}$ & $\begin{array}{l}0: 500 \\
0: 20: 120 \\
2 ; \\
\text { N/A; }\end{array}$ & $\begin{array}{l}\text { Atheros } 802.11 \\
\text { wireless cards }\end{array}$ \\
\hline$[58]$ & $\begin{array}{c}3100- \\
5300\end{array}$ & $\begin{array}{l}\text { Environmental } \\
\text { sensing system, } \\
3 \mathrm{G}, \quad 4 \mathrm{G}, \quad 5 \mathrm{G} \\
\text { networks, }\end{array}$ & $\begin{array}{l}\text { In open area } \\
\text { LOS COMM }\end{array}$ & $\mathrm{A} 2 \mathrm{G}$ & $\begin{array}{l}\text { PLE }=2.60-3.03 ; \\
\text { Shadow standard } \\
\text { deviation }=2.79- \\
5.30\end{array}$ & $\begin{array}{l}\text { CIR (PDP, } \\
\text { DDP, RMS-DS, } \\
\text { mean excess } \\
\text { delay, } \\
\text { coherence BW) } \\
\end{array}$ & $\begin{array}{l}5.6-16.5 \\
4: 4: 16 \\
1.5,0.07 \\
20\end{array}$ & $\begin{array}{l}\text { Time Domain P- } \\
410 \text { kit in bi-static } \\
\text { mode }\end{array}$ \\
\hline$[59]$ & $\begin{array}{c}900, \\
1800 \\
\text { and } \\
5000\end{array}$ & Search, rescue, & $\begin{array}{l}\text { LOS and } \\
\text { NLOS in } \\
\text { SISO and } \\
\text { beamforming } \\
(2 \times 1)\end{array}$ & $\mathrm{A} 2 \mathrm{G}$ & $\begin{array}{lr}\begin{array}{l}\text { LOS and } \\
\text { throughput }\end{array} & \text { vLOS } \\
2.22- & 30.59 \\
\text { Mbps. } & \end{array}$ & $\begin{array}{l}\text { DDP and } \\
\text { throughput }\end{array}$ & $\begin{array}{l}\text { 10:10:100; } \\
10,20,30 \\
1 ; \\
\text { N/A }\end{array}$ & $\begin{array}{l}\text { Spectrum } \\
\text { Analyzer, USRP }\end{array}$ \\
\hline$[60]$ & $\begin{array}{c}900, \\
1800 \\
\text { and } \\
5000\end{array}$ & $\begin{array}{l}\text { Search, rescue, } \\
\text { Disaster, military }\end{array}$ & $\begin{array}{l}\text { LOS in SISO } \\
\text { and } \\
\text { beamforming } \\
(2 \times 1) \text {, UAV } \\
\text { hovering and } \\
\text { encircling }\end{array}$ & A2G & \begin{tabular}{lr}
\multicolumn{2}{l}{ PLE $=0.07-1.99$. } \\
Shadow & standard \\
deviation $1.30-$ \\
6.12.
\end{tabular} & $\begin{array}{l}\text { DDP, BER and } \\
\text { throughput }\end{array}$ & $\begin{array}{l}10: 10: 100 \\
1 ; \\
10,20,30 \\
1,3,6\end{array}$ & $\begin{array}{l}\text { Spectrum } \\
\text { Analyzer, USRP }\end{array}$ \\
\hline$[61]$ & $\begin{array}{c}1817 \\
\text { and } \\
5760\end{array}$ & $\begin{array}{l}\text { Wildlife } \\
\text { Monitoring, } \\
\text { search and rescue }\end{array}$ & $\begin{array}{l}\text { In LOS } \\
\text { COMM, } \\
\text { UAV moving } \\
\text { and hovering }\end{array}$ & $\mathrm{A} 2 \mathrm{G}$ & $\begin{array}{l}\mathrm{PLE}=0.74,2.29 ; \\
\text { Shadow fading }= \\
1.23 \mathrm{~dB}, 2.15 \mathrm{~dB}\end{array}$ & $\begin{array}{l}\text { CIR } \quad \text { (PDP, } \\
\text { DDP, RMS-DS, } \\
\text { RKF) }\end{array}$ & $\begin{array}{l}210 ; \\
(20,30) \text { and }(0- \\
50) ; \\
\text { N/A; } \\
\text { N/A }\end{array}$ & USRP \\
\hline$[63]$ & $\begin{array}{c}3400- \\
3800\end{array}$ & $\begin{array}{l}\text { Disaster } \\
\text { management, } \\
\text { Cellular BS, Relay }\end{array}$ & $\begin{array}{l}\text { In LOS } \\
\text { COMM, } \\
\text { moving on } \\
\text { low altitudes }\end{array}$ & $\mathrm{A} 2 \mathrm{G}$ & $\begin{array}{l}\text { Modelled delay } \\
\text { parameters with } \\
\text { Rician, Weibull } \\
\text { and Lognormal } \\
\text { distributions. } \\
\end{array}$ & $\begin{array}{l}\text { CIR (PDP, } \\
\text { RMS-DS, mean } \\
\text { Depay Spread) }\end{array}$ & $\begin{array}{l}-10: 10 \\
5,10,15 \\
\approx 1.3 \\
0.5\end{array}$ & $\begin{array}{l}\text { Time domain } \\
\text { PulsON } 210 \text { kit }\end{array}$ \\
\hline [64] & 1200 & $\begin{array}{l}\text { Surveillance, } \\
\text { transportation, } \\
\text { disasters }\end{array}$ & Open area & $\mathrm{A} 2 \mathrm{G}$ & $\begin{array}{l}\text { RMS-DS }=294.78 \\
-286.20 \mathrm{~ns} .\end{array}$ & $\begin{array}{l}\text { CIR (PDP, RSS, } \\
\text { RMS-DS) }\end{array}$ & $\begin{array}{l}50 \\
4,10,40 \\
1.5 \\
0.5 \\
\end{array}$ & $\begin{array}{l}\text { Pulse based } \\
\text { channel sounder }\end{array}$ \\
\hline$[65]$ & $\begin{array}{c}785 \\
\text { and } \\
2160\end{array}$ & $\begin{array}{l}\text { Emergency based } \\
\text { COMM }\end{array}$ & $\begin{array}{l}\text { In LOS } \\
\text { COMM from } \\
\text { aerial ship (of } \\
\text { size } 35 \text { m) } \\
\begin{array}{l}\text { while } \\
\text { receiver on } \\
\text { vehicle }\end{array} \\
\end{array}$ & $\mathrm{A} 2 \mathrm{G}$ & $\begin{array}{l}\text { COST } 2100 \text { PL } \\
\text { model parameters. }\end{array}$ & $\begin{array}{l}\text { DDP, PDF of } \\
\text { shadow fading } \\
\text { throughput }\end{array}$ & $\begin{array}{l}0: 70000 ; \\
50, \quad 250, \quad 450, \\
715 \text { and } 950 ; \\
\text { Rx on vehicle; } \\
14 \quad \text { (speed of } \\
\text { vehicle); }\end{array}$ & $\begin{array}{l}\text { Frequency- } \\
\text { sweeping based } \\
\text { channel sounding }\end{array}$ \\
\hline$[66]$ & 5000 & $\begin{array}{l}\text { Emergency based } \\
\text { COMM, for safety } \\
\text { purposes }\end{array}$ & $\begin{array}{l}\text { In LOS } \\
\text { COMM from } \\
\text { UAV to car } \\
\text { on inclined } \\
\text { road }\end{array}$ & $\mathrm{A} 2 \mathrm{G}$ & $\begin{array}{l}\text { Modified } \\
\text { Gaussian fitting } \\
\text { with finding } \\
\text { goodness of fit. }\end{array}$ & $\begin{array}{l}\text { Packet delivery } \\
\text { ratio }\end{array}$ & $\begin{array}{l}0: 3000 \\
40,100 \\
\text { Rx on vehicle; }\end{array}$ & $\begin{array}{l}\text { Raspberry } \quad \mathrm{Pi}, \\
\text { Smartphone, } \\
\text { GRCBox }\end{array}$ \\
\hline$[67]$ & $\begin{array}{c}960- \\
977 \\
\text { and } \\
5000- \\
5150 \\
\end{array}$ & $\begin{array}{l}\text { Rescue, } \\
\text { surveillance, cargo }\end{array}$ & $\begin{array}{l}\text { Piloted } \\
\text { aircraft in } \\
\text { LOS COMM } \\
\text { over water }\end{array}$ & G2A & $\begin{array}{l}\text { Exponential } \\
\text { distribution on } \\
\text { time domain } \\
\text { parameters. }\end{array}$ & $\begin{array}{l}\text { CIR (PDP, } \\
\text { DDP, RMS-DS, } \\
\text { RKF) }\end{array}$ & $\begin{array}{l}2500 \\
4-20 \\
800 \\
90\end{array}$ & DSSS-CCS \\
\hline$[68]$ & $\begin{array}{c}968 \\
\text { and } \\
5060\end{array}$ & $\begin{array}{l}\text { Rescue, } \\
\text { surveillance, cargo }\end{array}$ & $\begin{array}{l}\text { Piloted } \\
\text { aircraft in } \\
\text { LOS COMM } \\
\text { in hills and } \\
\text { mountains } \\
\end{array}$ & G2A & PLE $=1.0-1.8$ & $\begin{array}{l}\text { CIR } \quad \text { (PDP, } \\
\text { DDP, RMS-DS, } \\
\text { RKF) }\end{array}$ & $\begin{array}{l}2500 \\
20 \\
1158,2728,3900 \\
75-95\end{array}$ & DSSS-CCS \\
\hline [69] & $\begin{array}{c}968 \\
\text { and } \\
5060\end{array}$ & $\begin{array}{l}\text { Rescue, } \\
\text { surveillance, cargo }\end{array}$ & $\begin{array}{l}\text { Piloted } \\
\text { aircraft in } \\
\text { LOS COMM } \\
\text { in sub-urban } \\
\text { and urban } \\
\end{array}$ & G2A & $\begin{array}{l}\text { Two ray modelling } \\
\text { parameters. }\end{array}$ & $\begin{array}{l}\text { CIR } \quad \text { PDP, } \\
\text { DDP, RMS-DS, } \\
\text { RKF) }\end{array}$ & $\begin{array}{l}542 ; \\
20 ; \\
1.07,1.69,20.31 \\
77\end{array}$ & DSSS-CCS \\
\hline$[70]$ & 2000 & $\begin{array}{l}\text { UAV enabled } \\
\text { wireless comm }\end{array}$ & $\begin{array}{l}\text { Tx on UAV } \\
\text { and } \mathrm{Rx} \text { on } \\
\text { moving car }\end{array}$ & $\mathrm{B} 2 \mathrm{~A}$ & $\begin{array}{l}\text { More adjacent cell } \\
\text { interference is } \\
\text { experienced in the } \\
\text { air than at ground. }\end{array}$ & $\begin{array}{l}\text { RSS, } \\
\text { Interference }\end{array}$ & $\begin{array}{l}\text { N/A; } \\
50,150 ; \\
\text { N/A; } \\
\text { UAV (5), car (5.5) }\end{array}$ & $\begin{array}{l}\text { Smart Phone App. } \\
\text { "TEMS Pocket } \\
\text { 16.3" }\end{array}$ \\
\hline [71] & 2585 & $\begin{array}{l}\text { Monitoring, } \\
\text { search and rescue, } \\
\text { farming, } \\
\text { transportation, } \\
\text { disaster } \\
\text { management }\end{array}$ & $\begin{array}{l}\text { LOS commin } \\
\text { the presence } \\
\text { of trees }\end{array}$ & $\mathrm{B} 2 \mathrm{~A}$ & $\begin{array}{l}\text { Deviations in large } \\
\text { scale fading } \\
\text { increases with } \\
\text { increase in altitude } \\
\text { or distance. }\end{array}$ & $\begin{array}{l}\text { CIR (PDP, RSS, } \\
\text { RMS-DS, RKF, } \\
\text { CDF, } \\
\text { shadowing) }\end{array}$ & $\begin{array}{l}100: 100: 500 \\
15,30,50,75,100 \\
20 \\
5.6,2.5\end{array}$ & USRP \\
\hline
\end{tabular}




\begin{tabular}{|c|c|l|l|l|l|l|l|l|}
\hline [72] & 800 & $\begin{array}{l}\text { Monitoring, } \\
\text { search and rescue, }\end{array}$ & $\begin{array}{l}\text { From BS to } \\
\text { UAV LOS } \\
\text { COMM }\end{array}$ & A2B & $\begin{array}{l}\text { PLE reduces with } \\
\text { increase of UAV } \\
\text { altitude in LOS } \\
\text { COMM. }\end{array}$ & $\begin{array}{l}\text { Altitude } \\
\text { dependent } \\
\text { exponent }\end{array}$ & $\begin{array}{l}\text { PL/A; } \\
\text { N/S; } \\
15,30,60,120 ; \\
4\end{array}$ \\
\hline
\end{tabular}

Table 3. Summary of simulation based channel modelling and relating parameters

\begin{tabular}{|c|c|c|c|c|c|c|c|c|}
\hline Ref & $\begin{array}{l}\text { Freq } \\
\text { MHz }\end{array}$ & Applications & Scenario & Type & Main Findings & $\begin{array}{l}\text { Studies } \\
\text { Parameter } \\
\text { S }\end{array}$ & $\begin{array}{l}\text { Distance }(\mathrm{m}) ; \\
\text { Tx height }(\mathrm{m}) ; \\
\text { Rx height }(\mathrm{m}) \\
\text { v }(\mathrm{m} / \mathrm{s})\end{array}$ & $\begin{array}{l}\text { Sounding } \\
\text { method } \\
\text { and/or } \\
\text { equipment }\end{array}$ \\
\hline [76] & $\begin{array}{c}2000- \\
6000\end{array}$ & $\begin{array}{l}\text { Cellular BS, } \\
\text { Disasters }\end{array}$ & $\begin{array}{l}\text { LOS and NLOS } \\
\text { with multipath } \\
\text { propagation }\end{array}$ & $\mathrm{A} 2 \mathrm{G}$ & $\begin{array}{lr}\text { Found ITU R- } \\
1410 \quad \text { model } \\
\text { parameters. }\end{array}$ & $\begin{array}{l}\text { Elevation } \\
\text { angle } \\
\text { dependent } \\
\text { probability of } \\
\text { LOS, } \\
\text { shadowing }\end{array}$ & $\begin{array}{l}211,000 ; \\
22,000 ; \\
\text { N/A; } \\
\text { N/A; }\end{array}$ & $\begin{array}{l}\text { N/A } \\
\text { (simulation } \\
\text { based) }\end{array}$ \\
\hline [77] & N/A & $\begin{array}{l}\text { Cellular BS, } \\
\text { Simulations based }\end{array}$ & $\begin{array}{l}\text { Assumes, all } \\
\text { MPCs lie in an } \\
\text { elliptical plane }\end{array}$ & $\mathrm{A} 2 \mathrm{G}$ & $\begin{array}{l}\text { Defined geometry- } \\
\text { based channel } \\
\text { model and found } \\
\text { Direction of } \\
\text { Arrival. } \\
\end{array}$ & $\begin{array}{l}\text { CIR (path } \\
\text { loss, delay } \\
\text { resolution, } \\
\text { DOA of } \\
\text { MPC) } \\
\end{array}$ & $\begin{array}{l}\text { N/A; } \\
\text { N/A; } \\
\text { N/A; } \\
\text { N/A; }\end{array}$ & $\begin{array}{l}\text { Derived } \\
\text { mathematical } \\
\text { relations }\end{array}$ \\
\hline [78] & $\begin{array}{l}200- \\
5000\end{array}$ & $\begin{array}{l}\text { Public safety, } \\
\text { disasters recovery, } \\
\text { military in the } \\
\text { field }\end{array}$ & $\begin{array}{l}\text { LOS, OLOS, } \\
\text { NLOS COMM } \\
\text { in hilly areas }\end{array}$ & $\mathrm{A} 2 \mathrm{G}$ & $\begin{array}{lr}\text { Eliminated } & \text { PLE } \\
\text { dependence } & \text { and } \\
\text { found mean } & \text { PL } \\
\text { parameters. } & \end{array}$ & $\begin{array}{l}\text { PED, CIR } \\
\text { (PDP), } \\
\text { Probability } \\
\text { (of LOS, } \\
\text { OLOS, } \\
\text { NLOS) }\end{array}$ & $\begin{array}{l}\text { N/A; } \\
100 ; \\
15 ; \\
\text { N/A; }\end{array}$ & $\begin{array}{l}\text { N/A } \\
\text { (ray tracing } \\
\text { simulations) }\end{array}$ \\
\hline [27] & $\begin{array}{c}700, \\
2000 \\
\text { and } \\
5800\end{array}$ & $\begin{array}{l}\text { Cellular BS, } \\
\text { disaster recovery, }\end{array}$ & $\begin{array}{l}\text { LOS COMM in } \\
\text { several } \\
\text { suburban and } \\
\text { urban use cases }\end{array}$ & $\mathrm{A} 2 \mathrm{G}$ & $\begin{array}{l}\text { Proposed a } \\
\text { statistical RPC } \\
\text { model for LAPs. }\end{array}$ & PED & $\begin{array}{l}\text { N/A } \\
200 ; \\
1.5 ; \\
\text { N/A }\end{array}$ & $\begin{array}{l}\text { N/A } \\
\text { (Wireless InSite } \\
\text { Simulator) }\end{array}$ \\
\hline [79] & 2442 & $\begin{array}{l}\text { Rescue, } \\
\text { surveillance, } \\
\text { weather detection, } \\
\text { monitoring } \\
\text { wildlife }\end{array}$ & $\begin{array}{l}\text { LOS and NLOS } \\
\text { COMM in } \\
\text { multipath } \\
\text { propagation for } \\
\text { high altitudes }\end{array}$ & A2G & $\begin{array}{l}\text { PLE is modelled } \\
\text { based on Tx } \\
\text { height. }\end{array}$ & $\begin{array}{l}\text { PED, } \\
\text { shadowing, } \\
\text { probability of } \\
\text { LOS }\end{array}$ & $\begin{array}{l}\text { N/A; } \\
\text { 100:100:2000 } \\
\text { N/A; } \\
\text { N/A; }\end{array}$ & $\begin{array}{l}\text { N/A } \\
\text { (Wireless InSite } \\
\text { Simulator) }\end{array}$ \\
\hline [80] & N/A & $\begin{array}{l}\text { Cellular BS, } \\
\text { disaster recovery, }\end{array}$ & $\begin{array}{l}\text { LOS and NLOS } \\
\text { COMM }\end{array}$ & $\mathrm{A} 2 \mathrm{G}$ & $\begin{array}{l}\text { Found the effect of } \\
\text { PLE and the } \\
\text { number of UAVs } \\
\text { on coverage } \\
\text { performance. }\end{array}$ & $\begin{array}{l}\text { Probability of } \\
\text { SNIR, area } \\
\text { spectral } \\
\text { efficiency }\end{array}$ & $\begin{array}{l}\text { N/A; } \\
\text { N/A; } \\
\text { N/A; } \\
\text { N/A; }\end{array}$ & N/A \\
\hline [81] & N/A & $\begin{array}{lr}\text { UAV } & \text { assisted } \\
\text { A2G } & \text { MIMO } \\
\text { COMM } & \end{array}$ & $\begin{array}{l}\text { Assumes, all } \\
\text { MPCs have } \\
\text { identical } \\
\text { propagation } \\
\text { delays coming } \\
\text { from the same } \\
\text { ellipsoid }\end{array}$ & $\mathrm{A} 2 \mathrm{G}$ & $\begin{array}{lr}\begin{array}{l}\text { Defined } \\
\text { cylinder }\end{array} & \text { bD } \\
\text { UAV } & \text { MIMO } \\
\text { channel model. }\end{array}$ & $\begin{array}{l}\text { spatial cross- } \\
\text { correlation } \\
\text { functions, } \\
\text { Doppler } \\
\text { power } \\
\text { spectrum } \\
\text { density, PDPs }\end{array}$ & $\begin{array}{l}\text { N/A; } \\
\text { N/A; } \\
\text { N/A; } \\
\text { N/A; }\end{array}$ & N/A \\
\hline [82] & N/A & $\begin{array}{lr}\text { UAV } & \text { assisted } \\
\text { A2G } & \text { MIMO } \\
\text { COMM } & \end{array}$ & $\begin{array}{l}\text { All MPCs } \\
\text { experience } \\
\text { similar delays } \\
\text { from UAV to } \\
\text { receiver. }\end{array}$ & $\mathrm{A} 2 \mathrm{G}$ & $\begin{array}{lr}\text { Defined } & 3 \mathrm{D} \\
\text { ellipsoid } & \text { channel } \\
\text { model. } & \end{array}$ & $\begin{array}{l}\text { spatial cross- } \\
\text { correlation } \\
\text { functions, } \\
\text { Doppler } \\
\text { power } \\
\text { spectrum } \\
\text { density, PDPs }\end{array}$ & $\begin{array}{l}\text { N/A; } \\
\text { N/A; } \\
\text { N/A; } \\
\text { N/A; }\end{array}$ & N/A \\
\hline [83] & $\begin{array}{l}2000 \\
5800 \\
5900\end{array}$ & $\begin{array}{lr}\text { Rescue } & \text { vehicles } \\
\text { for } & \text { disaster } \\
\text { management }\end{array}$ & LOS COMM & $\mathrm{G} 2 \mathrm{~A}$ & $\begin{array}{l}\text { Studied } \\
\text { connectivity } \\
\text { among UAV and } \\
\text { vehicles as a } \\
\text { function of UAV } \\
\text { altitude. }\end{array}$ & $\begin{array}{l}\text { Probability of } \\
\text { SNR greater } \\
\text { than a } \\
\text { threshold }\end{array}$ & $\begin{array}{l}\text { N/A; } \\
0: 1000 ; \\
\text { Vehicle height; } \\
\text { N/A; }\end{array}$ & $\begin{array}{l}\text { Derived } \\
\text { mathematical } \\
\text { relations }\end{array}$ \\
\hline [84] & N/A & $\begin{array}{l}\text { Comm from fixed } \\
\mathrm{BS} \text { to aerial and } \\
\text { ground } \mathrm{Rx}\end{array}$ & $\begin{array}{l}\text { LOS and NLOS } \\
\text { comm }\end{array}$ & $\mathrm{B} 2 \mathrm{~A}$ & $\begin{array}{l}\text { Limiting } \text { UAV } \\
\text { altitude, lowering } \\
\text { terrestrial BS } \\
\text { height and tilt } \\
\text { angle improves } \\
\text { COMM } \\
\text { performance. }\end{array}$ & $\begin{array}{l}\text { Coverage } \\
\text { probability } \\
\text { (UAV } \\
\text { altitude, BS } \\
\text { height, BS } \\
\text { antenna tilt) }\end{array}$ & $\begin{array}{l}\text { N/A; } \\
0: 120 \\
0: 50 \\
\text { N/A; }\end{array}$ & $\begin{array}{l}\text { N/A } \\
\text { (modified } \\
\text { Nakagami-m } \\
\text { fading model) }\end{array}$ \\
\hline
\end{tabular}




\section{Open Research Problems}

It is evident from the Table 22 and 3, the research work relating the RPC modelling for low altitude UAV enabled networks is still in an early stage. In addition, RPC modelling is mainly limited to the use cases in open areas with LOS communication links, very low altitudes, and provide very limited large scale and small scale fading analysis. Only a few of the measurements were comprehensively carried out (by modelling both large scale and small scale fading) however, they were limited to the use cases of open area and LOS links. It is important to perform further comprehensive RPC studies (measurements and simulation) for NLOS, relatively higher UAV altitudes, antenna orientation and polarization, characterization of shadowing due to UAV body and the use cases where UAV would be continuously flying while transmitting/receiving as well. In future wireless networks, UAV enabled wireless network coverage might be required in NLOS communication scenarios as well. Practically, there might be more chances of occurrence of scenarios having both the LOS and NLOS links due to the unexpected appearance of obstacles during UAV flights as a BS. For future perspective, UAVs will not only be used as a flying BS or relay. Instead, they will remain connected with everything $[47,86]$ i.e. Internet of Things (IoT), which for example may need to establish a communication link with indoor and outdoor electronic devices. This ultimately urges to investigate the RPC between a flying UAV and receiver(s) in various scenarios of indoor as well as outdoor e.g. receiver inside a building obstructed with of different materials and objects (e.g. wall and windows).

\section{Future Directions}

In addition to RPC modelling in UAV enabled wireless network, several other challenges need to be fulfilled to leverage the full benefits of flying BSs and relays in future wireless networks. This section particularly proposes the use cases inspired by the recommendations of standardization bodies for outdoor channel modelling [41-43,87-89] and limitation of the previous work (as discussed in section 2 and 3). In addition, this section discusses the challenges in UAV based 3D wireless networks, cellular connected UAVs and highlight the issues like UAV detection and battery power constraints.

\subsection{Proposed Use Cases}

Table 4 provides a summary of use cases which either have been investigated or still need to be investigated with some modification (if applicable). For better understanding, a colour scheme is used in Table 4. Four colours are used for four current statuses of summarized research from the literature: green (for the scenarios already done); yellow (for the scenarios need to be investigated with modifications e.g. in term of different altitudes); blue (for the scenarios yet to be investigated); and white (for the scenarios that are possibly not applicable).

Table 4 describes the scenarios in terms of various kind of UAV and receiver placement (receiver on ground/in vehicle and UAV moving or hovering) in different use cases e.g. open area, LOS, OLOS and NLOS for residential areas, industrial sites, high rise buildings, hilly area and in caves and tunnels. All these proposed scenarios tend to be significant for UAV enabled wireless network coverage not only for consumer and commercial market but also for emergency needs and disaster management.

Table 4. Use Cases for UAV based Channel Modelling with Research Gaps

SR: Static Receiver, RiV: Receiver in Vehicle, RoG: Receiver on Ground, AD: Already Done, Md: needs to be done with Modification, RI: required Investigation, NA: Not Applicable, OLOS: Obstructed LOS.

\begin{tabular}{|c|c|c|c|c|c|c|}
\hline \multirow{3}{*}{$\begin{array}{l}\text { Sr. } \\
\text { No }\end{array}$} & \multirow{3}{*}{ Use cases } & \multicolumn{5}{|c|}{ UAV and receiver placement } \\
\hline & & \multicolumn{2}{|c|}{$\begin{array}{c}\text { UAV } \\
\text { Hovering }\end{array}$} & \multirow{2}{*}{$\begin{array}{c}\text { Only } \\
\text { UAV } \\
\text { movi } \\
\text { ng } \\
\text { SR }\end{array}$} & \multicolumn{2}{|c|}{$\begin{array}{l}\text { Both UAV } \\
\text { and receiver } \\
\text { moving }\end{array}$} \\
\hline & & SR & $\mathbf{R i V}$ & & RoG & $\mathbf{R i V}$ \\
\hline 1 & Open Area & $\mathrm{AD}$ & RI & Md & RI & RI \\
\hline 2 & $\begin{array}{l}\text { OLOS and NLOS } \\
\text { in vegetation, } \\
\text { Halls, Residential } \\
\text { Areas, High rise } \\
\text { Buildings }\end{array}$ & Md & RI & Md & RI & RI \\
\hline 3 & $\begin{array}{l}\text { LOS, OLOS, and } \\
\text { NLOS in natural } \\
\text { disasters }\end{array}$ & RI & RI & RI & RI & RI \\
\hline 4 & $\begin{array}{l}\text { LOS, OLOS and } \\
\text { NLOS in } \\
\text { Industrial } \\
\text { catastrophes }\end{array}$ & RI & RI & RI & RI & RI \\
\hline 5 & $\begin{array}{l}\text { Caves and } \\
\text { tunnels } \\
\text { LOS, OLOS and } \\
\text { NLOS } \\
\end{array}$ & RI & RI & RI & RI & RI \\
\hline 6 & $\begin{array}{l}\text { LOS, OLOS and } \\
\text { NLOS in } \\
\text { While receiver on } \\
\text { various floors of } \\
\text { buildings }\end{array}$ & RI & NA & RI & RI & NA \\
\hline
\end{tabular}


Measurement campaigns can be initiated from the simplest use cases of open area to more focused and related use cases on several link-distances and the midband for $5 \mathrm{G}$ (preferably around $3.5 \mathrm{GHz}$ ) and the effects of natural disasters. These use cases should not be considered the only and hardly finalized, they can be adopted according to the latest recommendations or directions by standardization bodies or other stakeholders e.g. ITU, 3GPP, and GSMA.

\subsection{UAV Based Heterogeneous 3D Wireless Networks}

UAVs will be deployed as flying BS or relay for wireless coverage in 3D (three dimensional) future wireless networks [90,91]. Where they might be simultaneously connected with other flying BSs, terrestrial BSs, ground users, drones as user equipment and HAPs for backhaul. This kind of deployment of UAVs imparts the need for modelling the RPC among various kind of communication links. HAPs or satellites can play a vital role in establishing a link for backhaul. Researchers in [92], proposed a theoretical model to study 3D A2G propagation channel in terms of angle and time of arrival however required validation for measurements. Considering the 3GPP 3D channel model for terrestrial communication (i.e. LTE based), they can provide an initiative for a UAV enabled 3D channel models [93,94]. Furthermore, the placement of UAV BS for effective energy utilization with maximum coverage [95] while also taking into account the overall network delay [96], interference management from adjacent cells [97,98], dynamic spectrum access for UAV enabled networks [99], and 3D positing control [100] are vital research areas for UAV enabled network and required further investigation.

\subsection{Cellular Connected UAVs}

In the radio propagation channel modelling, it is intended that UAV will remain connected with a ground user as a BS or user equipment. Several other challenges exist for UAVs as flying BS. For example, estimating the number of UAV assisted BSs to provide wireless network coverage to a certain/uncertain number of ground users in a particular geographical area. Moreover, interested readers may refer to [62,84,101-104], for a detailed study of challenges expected to be faced in cellular connected UAVs for example the command and control of UAVs, defining combined network architecture for flying BSs and terrestrial $\mathrm{BSs}$, high data rate requirements, inter and intra cell interference mitigation, identification of a flying user equipment, determining optimal altitude of a UAV flying BS, an effective antenna pointing towards a ground user or terrestrial BS, enhanced mobility and effective handover with low latency. In addition, weather effects on the UAV enabled network, particularly in millimetre band, will be important to investigate like on-going research in $5 \mathrm{G}$ and B5G terrestrial networks [105].

\subsection{Other Challenges}

Other challenges in UAV enabled future wireless networks include e.g. detection and jamming of unauthorized UAVs [106-108], command and control of inter-connected UAVs [109,110], and battery or power constraint of the UAVs [111,112].

\section{CONCLUSION}

In addition to the continuously increasing utilization of UAVs in the consumer and commercial market, they are also now proposing to assist the existing terrestrial communication infrastructure for improved wireless network coverage. Particularly, the forthcoming 5G/B5G technologies are expected to provide improved wireless network coverage in the scenarios demanding high capacity and low latency on emergency needs, temporary coverage in hard to reach areas, IoT and for disasters management. It is expected that UAV enabled network will play an important role in future wireless networks to improve coverage and to provide on demand connectivity.

In this paper, a comprehensive survey of channel modelling for UAV enabled network has been presented for both measurement and simulation based approaches. In addition, potential open research problems are highlighted and proposed key use cases which will be vital for a functional low altitude UAV enable wireless networks particularly with the focus on the radio propagation channel modelling.

\section{Declaration of competing interest None}

\section{Acknowledgement}

Mr. Abrar Ahmad PhD work is funded by ViceChancellor Research Scholarship awarded by the Ulster University, UK 


\section{References}

[1] Recreational drone flights | UK Civil Aviation Authority, (n.d.). https://www.caa.co.uk/Consumers/Unmannedaircraft/Recreational-drones/Recreational-droneflights/ (accessed May 19, 2019).

[2] Federal Aviation Administration, Fact Sheet Small Unmanned Aircraft Regulations (Part 107), (2016).

https://www.faa.gov/news/fact_sheets/news_story. cfm?newsId=20516 (accessed May 20, 2019).

[3] Michał Mazur, Adam Wiśniewski, Clarity from above: PwC global report on the commercial applications of drone technology, (2016). https://www.pwc.pl/en/publikacje/2016/clarityfrom-above.html (accessed February 7, 2019).

[4] Drones for Commercial Applications | Tractica, (n.d.). https://www.tractica.com/research/dronesfor-commercial-applications/ (accessed August 20, 2019).

[5] P. Basu, J. Redi, V. Shurbanov, Coordinated flocking of UAVs for improved connectivity of mobile ground nodes, in: IEEE Mil. Commun. Conf., IEEE, 2004: pp. 1628-1634. doi:10.1109/MILCOM.2004.1495182.

[6] C.-M. Cheng, P.-H. Hsiao, H.T. Kung, D. Vlah, Maximizing Throughput of UAV-Relaying Networks with the Load-Carry-and-Deliver Paradigm, in: IEEE Wirel. Commun. Netw. Conf., IEEE, 2007: pp. 4417-4424. doi:10.1109/WCNC.2007.805.

[7] P. Zhan, K. Yu, A.L. Swindlehurst, Wireless relay communications with unmanned aerial vehicles: Performance and optimization, IEEE Trans. Aerosp. Electron. Syst. 47 (2011) 2068-2085. doi:10.1109/TAES.2011.5937283.

[8] İ. Bekmezci, O.K. Sahingoz, Ş. Temel, Flying AdHoc Networks (FANETs): A survey, Ad Hoc Networks. $\quad 11 \quad$ (2013) 1254-1270. doi:10.1016/J.ADHOC.2012.12.004.

[9] Y. Zeng, R. Zhang, T.J. Lim, Wireless communications with unmanned aerial vehicles: Opportunities and challenges, IEEE Commun. Mag. 54 (2016) 36-42. doi:10.1109/MCOM.2016.7470933.

[10] M. Mozaffari, W. Saad, M. Bennis, M. Debbah, Mobile Unmanned Aerial Vehicles (UAVs) for Energy-Efficient Internet of Things Communications, IEEE Trans. Wirel. Commun. 16 (2017) 7574-7589. doi:10.1109/TWC.2017.2751045.

[11] S. Sekander, H. Tabassum, E. Hossain, Multi-Tier Drone Architecture for 5G/B5G Cellular Networks:
Challenges, Trends, and Prospects, IEEE Commun. Mag. $56 \quad$ (2018) 96-103. doi:10.1109/MCOM.2018.1700666.

[12] M. Mozaffari, W. Saad, M. Bennis, Y.-H. Nam, M. Debbah, A Tutorial on UAVs for Wireless Networks: Applications, Challenges, and Open Problems, IEEE Commun. Surv. Tutorials. 21 (2019) 2334-2360. doi:10.1109/COMST.2019.2902862.

[13] Y. Zhou, N. Cheng, N. Lu, X.S. Shen, Multi-UAVAided Networks: Aerial-Ground Cooperative Vehicular Networking Architecture, IEEE Veh. Technol. Mag. $10 \quad$ (2015) 36-44. doi:10.1109/MVT.2015.2481560.

[14] M. Erdelj, E. Natalizio, K.R. Chowdhury, I.F. Akyildiz, Help from the Sky: Leveraging UAVs for Disaster Management, IEEE Pervasive Comput. 16 (2017) 24-32. doi:10.1109/MPRV.2017.11.

[15] Guang Yang, Xingqin Lin, Yan Li, Hang Cui, Min Xu, Dan Wu, Henrik Rydén, Sakib Bin Redhwan, A Telecom Perspective on the Internet of Drones: From LTE-Advanced to 5G, in: ArXiv:1803.11048, arxiv.org, 2018. https://arxiv.org/ftp/arxiv/papers/1803/1803.11048 .pdf (accessed November 30, 2018).

[16] G. Baldini, S. Karanasios, D. Allen, F. Vergari, Survey of Wireless Communication Technologies for Public Safety, IEEE Commun. Surv. Tutorials. 16 (2014) 619-641. doi:10.1109/SURV.2013.082713.00034.

[17] M. Kobayashi, Experience of infrastructure damage caused by the Great East Japan Earthquake and countermeasures against future disasters, IEEE Commun. Mag. $52 \quad$ (2014) 23-29. doi:10.1109/MCOM.2014.6766080.

[18] H. Ritchie, M. Roser, Natural Disasters - Empirical View, Our World Data. (2019). https://ourworldindata.org/natural-disasters (accessed May 22, 2019).

[19] M. Casoni, C.A. Grazia, M. Klapez, N. Patriciello, A. Amditis, E. Sdongos, Integration of satellite and LTE for disaster recovery, IEEE Commun. Mag. 53 (2015) 47-53. doi:10.1109/MCOM.2015.7060481.

[20] B.S. Manoj, A.H. Baker, Communication challenges in emergency response, Commun. $\begin{array}{llll}\text { ACM. } & 50 & \text { (2007) }\end{array}$ doi:10.1145/1226736.1226765.

[21] S. Waharte, N. Trigoni, Supporting Search and Rescue Operations with UAVs, in: Int. Conf. Emerg. Secur. Technol., 2010: pp. 142-147. doi:10.1109/EST.2010.31.

[22] S. Kandeepan, K. Gomez, T. Rasheed, L. Reynaud, Energy efficient cooperative strategies in hybrid aerial-terrestrial networks for emergencies, in: 
IEEE 22nd Int. Symp. Pers. Indoor Mob. Radio Commun., IEEE, 2011: pp. 294-299. doi:10.1109/PIMRC.2011.6139969.

[23] A. Thomas, G. Raja, FINDER: A D2D based critical communications framework for disaster management in 5G, Peer-to-Peer Netw. Appl. (2018) 1-12. doi:10.1007/s12083-018-0689-2.

[24] A.K. Widiawan, R. Tafazolli, High Altitude Platform Station (HAPS): A Review of New Infrastructure Development for Future Wireless Communications, Wirel. Pers. Commun. 42 (2007) 387-404. doi:10.1007/s11277-006-9184-9.

[25] T. Rasheed, K. Gomez, I. Bucaille, ABSOLUTE Aerial Base Stations with Opportunistic Links for Unexpected \& Temporary Events, Second Rep. Dissem. Act. (2014). https://cordis.europa.eu/docs/projects/cnect/2/3186 32/080/deliverables/001-

FP7ICT20118318632ABSOLUTED813v4Ares20 144050905.pdf (accessed November 28, 2018).

[26] K. Gomez, Kandeepan Sithamparanathan, Macià Vidal, Vincent Boussemart, Raquel Ramos, Romain Hermenier, Tinku Rasheed, Leonardi Goratti, Laurent Reynaud, David Grace, Qiyang Zhao, Yunbo Han, Salahedin Rehan, Nils Morozs, Tao Jiang, Isabelle Bucaille, Tom Wirth, Roberta Campo, Tomaž Javornik, Aerial base stations with opportunistic links for next generation emergency communications, IEEE Commun. Mag. 54 (2016) 31-39. doi:10.1109/MCOM.2016.7452263.

[27] A. Al-hourani, S. Kandeepan, A. Jamalipour, Modeling Air-to-Ground Path Loss for Low Altitude Platforms in Urban Environments, in: Globecom 2014 - Symp. Sel. Areas Commun., IEEE, 2014: pp. 2898-2904. doi:10.1109/GLOCOM.2014.7037248.

[28] S. Kandeepan, K. Gomez, L. Reynaud, T. Rasheed, Aerial-terrestrial communications: terrestrial cooperation and energy-efficient transmissions to aerial base stations, IEEE Trans. Aerosp. Electron. $\begin{array}{llll}\text { Syst. } & 50 & \text { (2014) }\end{array}$ doi:10.1109/TAES.2014.130012.

[29] S.F.F.M.A. (FSD), Drones in humanitarian action: a guide to the use of airborne systems in humanitarian crises, (2016) 59. http://drones.fsd.ch/wpcontent/uploads/2016/11/Drones-in-HumanitarianAction.pdf (accessed December 2, 2018).

[30] K. Gomez, T. Rasheed, L. Reynaud, I. Bucaille, Realistic deployments of LTE-based Hybrid Aerial-Terrestrial Networks for public safety, in: 18th IEEE Int. Work. Comput. Aided Model. Des. Commun. Links Networks, IEEE, 2013: pp. 233237. doi:10.1109/CAMAD.2013.6708123.
[31] A. Merwaday, A. Tuncer, A. Kumbhar, I. Guvenc, Improved throughput coverage in natural disasters: Unmanned aerial base stations for public-safety communications, IEEE Veh. Technol. Mag. 11 (2016) 53-60. doi:10.1109/MVT.2016.2589970.

[32] M. Gapeyenko, I. Bor-Yaliniz, S. Andreev, H. Yanikomeroglu, Y. Koucheryavy, Effects of blockage in deploying mmWave drone base stations for $5 \mathrm{~g}$ networks and beyond, in: IEEE Int. Conf. Commun. Work., IEEE, 2018: pp. 1-6. doi:10.1109/ICCW.2018.8403671.

[33] E. Vinogradov, H. Sallouha, S. De Bast, M.M. Azari, S. Pollin, Tutorial on UAVs: A Blue Sky View onWireless Communication, ArXiv Prepr. ArXiv1901.02306.

(2019). doi:10.13052/jmm1550-4646.1443.

[34] N. Zhao, W. Lu, M. Sheng, Y. Chen, J. Tang, F.R. $\mathrm{Yu}, \mathrm{K} . \mathrm{K}$. Wong, UAV-Assisted Emergency Networks in Disasters, IEEE Wirel. Commun. 26 (2019) 45-51. doi:10.1109/MWC.2018.1800160.

[35] G. Tuna, B. Nefzi, G. Conte, Unmanned aerial vehicle-aided communications system for disaster recovery, J. Netw. Comput. Appl. 41 (2014) 27-36. doi:10.1016/j.jnca.2013.10.002.

[36] GSMA, Mobile-Enabled Unmanned Aircraft Aircraft, (2018). https://www.gsma.com/iot/wpcontent/uploads/2018/02/Mobile-Enabled-

Unmanned-Aircraft-web.pdf (accessed February 14, 2019).

[37] M. Asadpour, B. Van den Bergh, D. Giustiniano, K.A. Hummel, S. Pollin, B. Plattner, Micro Aerial Vehicle Networks: An Experimental Analysis of Challenges and Opportunities, IEEE Commun. Mag. $\quad 52 \quad$ (2014) 141-149. doi:10.1109/MCOM.2014.6852096.

[38] Qualcomm Technologies, Spectrum for 4G and 5G, Qualcomm Technol. Inc. (2017). https://www.qualcomm.com/media/documents/file s/spectrum-for-4g-and-5g.pdf (accessed February 14, 2019).

[39] F.C. Commission, Before the Federal Communications Commission Washington, D.C. 20554, (2002). https://transition.fcc.gov/Bureaus/Engineering_Te chnology/Orders/2002/fcc02048.pdf (accessed November 21, 2018).

[40] $4 \mathrm{G}$ frequency bands - Which UK networks will my phone work on?, (n.d.). https://www.4g.co.uk/4gfrequencies-uk-need-know/ (accessed November 21, 2018).

[41] 3GPP TSG RAN WG1, Requirements of Connectivity Services for Drones (R1-1704429), (2017).

https://portal.3gpp.org/ngppapp/TdocList.aspx?me 
etingId=17061 (accessed February 7, 2019).

3GPP TSG RAN WG1, Evaluation Scenarios and Channel Models for Drones (R1-1704430), (2017). https://portal.3gpp.org/ngppapp/TdocList.aspx?me etingId=17061 (accessed February 7, 2019).

[43] ITU-Technical Paper, Use cases and scenarios for disaster information service using unmanned aerial vehicles, (2018). https://www.itu.int/dms_pub/itut/opb/tut/T-TUT-DIS-2018-UAV-PDF-E.pdf (accessed February 11, 2019).

[44] C. Yan, L. Fu, J. Zhang, J. Wang, A Comprehensive Survey on UAV Communication Channel Modeling, IEEE Access. 7 (2019) 107769-107792. doi:10.1109/ACCESS.2019.2933173.

[45] I. Bor-Yaliniz, H. Yanikomeroglu, The New Frontier in RAN Heterogeneity: Multi-Tier DroneCells, IEEE Commun. Mag. 54 (2016) 48-55. doi:10.1109/MCOM.2016.1600178CM.

[46] D.W. Matolak, Air-Ground Channels \& Models: Comprehensive Review and Considerations for Unmanned Aircraft Systems, in: IEEE Aerosp. Conf., IEEE, 2012: pp. 1-17. doi:10.1109/AERO.2012.6187152.

[47] N. Hossein Motlagh, T. Taleb, O. Arouk, LowAltitude Unmanned Aerial Vehicles-Based Internet of Things Services: Comprehensive Survey and Future Perspectives, IEEE Internet Things J. 3 (2016) 899-922. doi:10.1109/JIOT.2016.2612119.

[48] Y. Li, L. Cai, UAV-Assisted Dynamic Coverage in a Heterogeneous Cellular System, IEEE Netw. 31 (2017) 56-61. doi:10.1109/MNET.2017.1600280.

[49] Aziz Altaf Khuwaja, Y. Chen, N. Zhao, M.-S. Alouini, P. Dobbins, A Survey of Channel Modeling for UAV Communications, IEEE Commun. Surv. Tutorials. 20 (2018) 2804-2821. doi:10.1109/COMST.2018.2856587.

[50] W. Khawaja, I. Guvenc, D.W. Matolak, U. Fiebig, N. Schneckenberger, A Survey of Air-to-Ground Propagation Channel Modeling for Unmanned Aerial Vehicles, IEEE Commun. Surv. Tutorials. 21 (2019) 2361-2391. doi:10.1109/COMST.2019.2915069.

[51] S. Mignardi, C. Buratti, A. Bazzi, R. Verdone, Trajectories and resource management of flying base stations for C-V2X, Sensors (Switzerland). 19 (2019). doi:10.3390/s19040811.

[52] A. F.Molisch, Wireless communications, second Edi, John Wiley \& Sons Ltd., 2011. doi:10.1002/9781119992806.fmatter.

[53] John David Parsons, The Mobile Radio Propagation Channel, Second, John Wiley \& Sons Ltd., 2000. http://www.wiley.co.uk (accessed February 26, 2019).

[54] I. Oppermann, M. Hämäläinen, J. Iinatti, UWB theory and applications, Wiley, 2004. https://books.google.co.uk/books?hl=en\&lr=\&id= KiaquNK9r4oC\&oi=fnd\&pg=PR7\&dq $=\mathrm{UWB}+\mathrm{ti}$ me+domain+sounder\&ots=rZWucttzv $9 \&$ sig $=w 7 a$ vJ6AG305ZdwoiIVCtKXbE9SI\#v=onepage \&q\&f =false (accessed March 29, 2019).

[55] S. Salous, Radio Propagation Measurement and Channel Modelling, John Wiley \& Sons, 2013.

[56] R. Verdone, A. Zanella, Pervasive mobile and ambient wireless communications : COST Action 2100, Springer, 2012. doi:10.1007/978-1-44712315-6.

[57] E. Yanmaz, R. Kuschnig, C. Bettstetter, Channel measurements over 802.11a-based UAV-to-ground links, in: IEEE GLOBECOM Work. (GC Wkshps), IEEE, 2011: pp. 1280-1284. doi:10.1109/GLOCOMW.2011.6162389.

[58] W. Khawaja, I. Guvenc, D. Matolak, UWB channel sounding and modeling for UAV air-to-ground propagation channels, in: IEEE Glob. Commun. Conf., IEEE, USA, 2016. doi:10.1109/GLOCOM.2016.7842372.

[59] Y. Shi, R. Enami, J. Wensowitch, J. Camp, Measurement-Based Characterization of LOS and NLOS Drone-to-Ground Channels, in: IEEE Wirel. Commun. Netw. Conf., IEEE, 2018: pp. 1-6. https://ieeexplore.ieee.org/stamp/stamp.jsp?tp=\&a rnumber=8377104.

[60] Y. Shi, R. Enami, J. Wensowitch, J. Camp, UABeam: UAV-based beamforming system analysis with in-field air-to-ground channels, in: 15th Annu. IEEE Int. Conf. Sensing, Commun. Netw., IEEE, 2018: pp. 1-9. doi:10.1109/SAHCN.2018.8397110.

[61] X. Cai, A. Gonzalez-plaza, D. Alonso, L. Zhang, C.B. Rodríguez, A.P. Yuste, Low Altitude UAV Propagation Channel Modelling, in: 11th Eur. Conf. Antennas Propag., IEEE, 2017: pp. 14431447. doi:10.23919/EuCAP.2017.7928479.

[62] X. Lin, V. Yajnanarayana, S.D. Muruganathan, S. Gao, H. Asplund, H.-L. Maattanen, M. Bergstrom, S. Euler, Y.-P.E. Wang, The Sky Is Not the Limit: LTE for Unmanned Aerial Vehicles, IEEE Commun. Mag. 56 (2018) 204-210. doi:10.1109/MCOM.2018.1700643.

[63] P. Catherwood, B. Black, E.B. Mohamed, A.A. Cheema, J. McLaughlin, Radio Channel Characterization of Mid-band 5G Service Delivery for Ultra-Low Altitude Aerial Base Stations, IEEE Access. (2018). https://pure.ulster.ac.uk/en/publications/radiochannel-characterization-of-mid-band-5g-servicedelivery-fo (accessed January 11, 2019).

[64] C. Calvo-Ramírez, A. Gonzalez-Plaza, C. Briso, A. 
Paez-Yuste, Wide band propagation measurements and modelling for low altitude UAVs, in: 12th Eur. Conf. Antennas Propag., Institution of Engineering and Technology, 2018. doi:10.1049/cp.2018.0947.

[65] R. Zhang, Q. Guo, D. Zhai, D. Zhou, X. Du, M. Guizani, Channel Measurement and Resource Allocation Scheme for Dual-Band Airborne Access Networks, IEEE Access. 7 (2019) 80870-80883. doi:10.1109/ACCESS.2019.2923538.

[66] S.A. Hadiwardoyo, E. Hernández-Orallo, C.T. Calafate, J.C. Cano, P. Manzoni, Experimental characterization of UAV-to-car communications, Comput. Networks. 136 (2018) 105-118. doi:10.1016/j.comnet.2018.03.002.

[67] D.W. Matolak, R. Sun, Air-Ground Channel Characterization for Unmanned Aircraft SystemsPart I: Methods, Measurements, and Models for Over-Water Settings, IEEE Trans. Veh. Technol. 66 (2017) 26-44. doi:10.1109/TVT.2016.2530306.

[68] R. Sun, D.W. Matolak, Air-Ground Channel Characterization for Unmanned Aircraft Systems Part II: Hilly and Mountainous Settings, IEEE Trans. Veh. Technol. 66 (2017) 1913-1925. doi:10.1109/TVT.2016.2585504.

[69] D.W. Matolak, R. Sun, Air-Ground Channel Characterization for Unmanned Aircraft SystemsPart III: The Suburban and Near-Urban Environments, IEEE Trans. Veh. Technol. 66 (2017) 6607-6618. doi:10.1109/TVT.2017.2659651.

[70] X. Lin, R. Wiren, S. Euler, A. Sadam, H.-L. Maattanen, S.D. Muruganathan, S. Gao, Y.-P.E. Wang, J. Kauppi, Z. Zou, V. Yajnanarayana, Mobile Networks Connected Drones: Field Trials, Simulations, and Design Insights, ArXiv:1801.10508. (2018). https://arxiv.org/abs/1801.10508 (accessed November 30, 2018).

[71] X. Cai, J. Rodriguez-Pineiro, X. Yin, N. Wang, B. Ai, G.F. Pedersen, A. Perez, L. Tian, An Empirical Air-to-Ground Channel Model Based on Passive Measurements in LTE, IEEE Trans. Veh. Technol. 68 (2019) 1-1. doi:10.1109/TVT.2018.2886961.

[72] R. Amorim, H. Nguyen, P. Mogensen, I.Z. Kovacs, J. Wigard, T.B. Sorensen, Radio Channel Modeling for UAV Communication Over Cellular Networks, IEEE Wirel. Commun. Lett. 6 (2017) 514-517. doi:10.1109/LWC.2017.2710045.

[73] J. Kosmerl, A. Vilhar, Base stations placement optimization in wireless networks for emergency communications, in: IEEE Int. Conf. Commun. Work., IEEE, 2014: pp. 200-205. doi:10.1109/ICCW.2014.6881196.

[74] A. Borhani, M. Ptzold, Modeling of vehicle-to- vehicle channels in the presence of moving scatterers, in: IEEE Veh. Technol. Conf., IEEE, 2012. doi:10.1109/VTCFall.2012.6398895.

[75] A.F. Molisch, F. Tufvesson, J. Karedal, C.F. Mecklenbräuker, A survey on vehicle-to-vehicle propagation channels, IEEE Wirel. Commun. 16 (2009) 12-22. doi:10.1109/MWC.2009.5361174.

[76] J. Holis, P. Pechac, Elevation Dependent Shadowing Model for Mobile Communications via High Altitude Platforms in Built-Up Areas, IEEE Trans. Antennas Propag. 56 (2008) 1078-1084. doi:10.1109/TAP.2008.919209.

[77] W.G. Newhall, J.H. Reed, A GEOMETRIC AIRTO-GROUND RADIO CHANNEL MODEL, in: IEEE Mil. Commun. Conf., IEEE, California, 2002: $\quad$ pp. 632-636. doi:10.1109/MILCOM.2002.1180518.

[78] Qixing Feng, J. McGeehan, E.K. Tameh, A.R. Nix, Path Loss Models for Air-to-Ground Radio Channels in Urban Environments, in: IEEE 63rd Veh. Technol. Conf., 2006: pp. 2901-2905. doi:10.1109/VETECS.2006.1683399.

[79] N. Sharma, M. Magarini, L. Dossi, L. Reggiani, R. Nebuloni, A study of channel model parameters for aerial base stations at $2.4 \mathrm{GHz}$ in different environments, in: IEEE Annu. Consum. Commun. Netw. Conf., IEEE, 2018. doi:10.1109/CCNC.2018.8319165.

[80] E. Turgut, M.C. Gursoy, Downlink Analysis in Unmanned Aerial Vehicle (UAV) Assisted Cellular Networks With Clustered Users, IEEE Access. 6 (2018) 36313-36324. doi:10.1109/ACCESS.2018.2841655.

[81] H. Jiang, Z. Zhang, L. Wu, J. Dang, ThreeDimensional Geometry-Based UAV-MIMO Channel Modeling for A2G Communication Environments, IEEE Commun. Lett. 22 (2018) 1438-1441. doi:10.1109/LCOMM.2018.2828110.

[82] H. Jiang, Z. Zhang, G. Gui, Three-Dimensional Non-Stationary Wideband Geometry-Based UAV Channel Model for A2G Communication Environments, IEEE Access. 7 (2019) 2611626122. doi:10.1109/ACCESS.2019.2897431.

[83] S. Jia, L. Zhang, Modelling unmanned aerial vehicles base station in ground-to-air cooperative networks, IET Commun. 11 (2017) 1187-1194. doi:10.1049/iet-com.2016.0808.

[84] M.M. Azari, F. Rosas, A. Chiumento, S. Pollin, Coexistence of Terrestrial and Aerial Users in Cellular Networks, in: IEEE Globecom Work. GC Wkshps 2017 - Proc., IEEE, 2017: pp. 1-6. doi:10.1109/GLOCOMW.2017.8269068.

[85] T.S. Rappaport, S. Sun, M. Shafi, Investigation and Comparison of 3GPP and NYUSIM Channel 
Models for 5G Wireless Communications, in: IEEE 86th Veh. Technol. Conf., IEEE, 2017: pp. 1-5. doi:10.1109/VTCFall.2017.8287877.

[86] X. Zhou, S. Durrani, J. Guo, H. Yanikomeroglu, Underlay Drone Cell for Temporary Events: Impact of Drone Height and Aerial Channel Environments, IEEE Internet Things J. 6 (2019) 1704-1718. doi:10.1109/JIOT.2018.2875166.

[87] K. Haneda, J. Zhang, L. Tian, G. Liu, Y. Zheng, H. Asplund, J. Li, Y. Wang, D. Steer, C. Li, T. Balercia, S. Lee, Y. Kim, A. Ghosh, T. Thomas, T. Nakamura, Y. Kakishima, T. Imai, H. Papadopoulas, T.S. Rappaport, G.R.J. Maccartney, M.K. Samimi, S. Sun, O. Koymen, S. Hur, J. Park, C. Zhang, E. Mellios, A.F. Molisch, S.S. Ghassemzadeh, A. Ghosh, 5G 3GPP-like Channel Models for Outdoor Urban Microcellular and Macrocellular Environments, in: IEEE 83rd Veh. Technol. Conf. (VTC Spring), 2016. doi:10.1109/VTCSpring.2016.7503971.

[88]

I. Radiocommunication Bureau, RECOMMENDATION ITU-R P.1411-6 Propagation data and prediction methods for the planning of short-range outdoor radiocommunication systems and radio local area networks in the frequency range $300 \mathrm{MHz}$ to 100 GHz, 2012. http://www.itu.int/ITU$\mathrm{R} /$ go/patents/en (accessed November 21, 2019).

[89] ITU, Studies for short-path propagation data and models for terrestrial radiocommunication systems in the frequency range $6 \mathrm{GHz}$ to $100 \mathrm{GHz}$ (Rep. ITU-R P.2406-0), (2017). http://www.itu.int/ITUR/go/patents/en (accessed February 8, 2019).

[90] M. Mozaffari, A.T.Z. Kasgari, W. Saad, M. Bennis, M. Debbah, Beyond 5G with UAVs: Foundations of a 3D Wireless Cellular Network, IEEE Trans. Wirel. Commun. 18 (2019) 357-372. doi:10.1109/TWC.2018.2879940.

[91] Y. Zeng, J. Lyu, R. Zhang, Cellular-connected UAV: Potential, challenges, and promising technologies, IEEE Wirel. Commun. 26 (2019) 120-127. doi:10.1109/MWC.2018.1800023.

[92] S.M. Gulfam, S.J. Nawaz, A. Ahmed, M.N. Patwary, Angle and time of arrival characteristics of 3D air-to-ground radio propagation environments, Comput. Commun. 112 (2017) 22 37. doi:10.1016/j.comcom.2017.08.011.

[93] Y.H. Nam, Y. Li, J.C. Zhang, 3D channel models for elevation beamforming and FD-MIMO in LTEA and 5G, in: Conf. Rec. - Asilomar Conf. Signals, Syst. Comput., IEEE Computer Society, 2015: pp. 805-809. doi:10.1109/ACSSC.2014.7094561.

[94] 3GPP TR 36.873 V1.3.0 (Release 12), Study on 3D channel model for LTE, 2014.
[95] M. Alzenad, A. El-Keyi, F. Lagum, H. Yanikomeroglu, 3-D Placement of an Unmanned Aerial Vehicle Base Station (UAV-BS) for EnergyEfficient Maximal Coverage, IEEE Wirel. Commun. Lett. 6 (2017) 434-437. doi:10.1109/LWC.2017.2700840.

[96] V. Sharma, R. Sabatini, S. Ramasamy, UAVs assisted delay optimization in heterogeneous wireless networks, IEEE Commun. Lett. 20 (2016) 2526-2529. doi:10.1109/LCOMM.2016.2609900.

[97] M. Jacovic, O. Bshara, K.R. Dandekar, Waveform Design of UAV Data Links in Urban Environments for Interference Mitigation, in: IEEE 88th Veh. Technol. Conf., IEEE, 2018: pp. 1-5. doi:10.1109/VTCFall.2018.8690581.

[98] V. Yajnanarayana, Y.-P. Eric Wang, S. Gao, S. Muruganathan, X. Lin Ericsson, Interference Mitigation Methods for Unmanned Aerial Vehicles Served by Cellular Networks, in: IEEE 5G World Forum, IEEE, 2018: pp. 118-122. doi:10.1109/5GWF.2018.8517087.

[99] A.A. Cheema, S. Salous, Spectrum Occupancy Measurements and Analysis in $2.4 \mathrm{GHz}$ WLAN, $\begin{array}{lll}\text { Electronics. } & 8 & \text { (2019). }\end{array}$ doi:10.3390/electronics8091011.

[100] L. Shan, R. Miura, T. Kagawa, F. Ono, H.-B. Li, F. Kojima, Machine Learning-Based Field Data Analysis and Modeling for Drone Communications, IEEE Access. 7 (2019) 7912779135. doi:10.1109/ACCESS.2019.2922544.

[101] B. Van Der Bergh, A. Chiumento, S. Pollin, LTE in the sky: trading off propagation benefits with interference costs for aerial nodes, IEEE Commun. Mag. $\quad 54 \quad$ (2016) 44-50. doi:10.1109/MCOM.2016.7470934.

[102] M.M. Azari, F. Rosas, S. Pollin, Reshaping Cellular Networks for the Sky: Major Factors and Feasibility, in: IEEE Int. Conf. Commun., Institute of Electrical and Electronics Engineers Inc., 2018. doi:10.1109/ICC.2018.8422685.

[103] U. Challita, W. Saad, C. Bettstetter, CellularConnected UAVs over 5G: Deep Reinforcement Learning for Interference Management, ArXiv Prepr. ArXiv1801.05500. (2018). doi:10.1109/TWC.2019.2900035.

[104] A. Fakhreddine, C. Bettstetter, S. Hayat, R. Muzaffar, D. Emini, Handover Challenges for Cellular-Connected Drones, in: Proc. 5th Work. Micro Aer. Veh. Networks, Syst. Appl., Association for Computing Machinery (ACM), 2019: pp. 9-14. doi:10.1145/3325421.3329770.

[105] J. Huang, Y. Cao, X. Raimundo, A. Cheema, S. Salous, Rain Statistics Investigation and Rain Attenuation Modeling for Millimeter Wave Short- 
Range Fixed Links, IEEE Access. 7 (2019) 156110-156120. doi:10.1109/ACCESS.2019.2949437.

[106] J. Farlik, M. Kratky, J. Casar, Detectability and jamming of small UAVs by commercially available low-cost means, in: IEEE Int. Conf. Commun., Institute of Electrical and Electronics Engineers Inc., 2016: pp. 327-330. doi:10.1109/ICComm.2016.7528287.

[107] K. Parlin, M.M. Alam, Y. Le Moullec, Jamming of UAV remote control systems using software defined radio, in: Int. Conf. Mil. Commun. Inf. Syst. ICMCIS, Institute of Electrical and Electronics Engineers Inc., 2018: pp. 1-6. doi:10.1109/ICMCIS.2018.8398711.

[108] I. Guvenc, F. Koohifar, S. Singh, M.L. Sichitiu, D. Matolak, Detection, Tracking, and Interdiction for Amateur Drones, IEEE Commun. Mag. 56 (2018) 75-81. doi:10.1109/MCOM.2018.1700455.

[109] J. Elston, E. Frew, B. Argrow, Networked UAV command, control and communication, in: Collect. Tech. Pap. - AIAA Guid. Navig. Control Conf. Exibit, 2006. doi:10.2514/6.2006-6465.

[110] K. Mathia, G. Lafferriere, T. Titensor, Cooperative Control of UAV Platoons-A Prototype, in: Euro UAV 2007 Conf. Exhib., Paris, France, 2007. https://www.researchgate.net/publication/2286400 86 (accessed October 30, 2019).

[111] T. Campi, S. Cruciani, M. Feliziani, F. Maradei, High efficiency and lightweight wireless charging system for drone batteries, in: AEIT Int. Annu. Conf., IEEE, 2017: pp. 1-6. doi:10.23919/AEIT.2017.8240539.

[112] A. Rohan, M. Rabah, F. Asghar, M. Talha, S.-H. Kim, Advanced Drone Battery Charging System, J. Electr. Eng. Technol. 14 (2019) 1395-1405. doi:10.1007/s42835-019-00119-8.

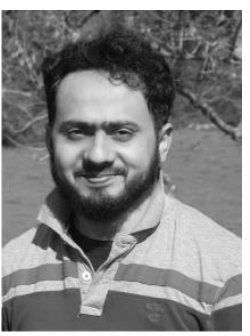

Abrar Ahmad received the B.E. degree in Electronics Engineering from COMSATS Abbottabad, Pakistan in 2012 and the M.S. degree in Electrical Engineering in 2016 from Institute of Space Technology (IST), Islamabad, Pakistan. In 2016, he started his career in teaching by joining MY University, Islamabad, Pakistan as a Lecturer in the Department of Electrical Engineering and in March 2018, he joined IST Islamabad, as a Lecturer.

Currently, he is doing PhD in Electrical Engineering from the Ulster University, Jordanstown, UK, funded by ViceChancellor's Research Scholarship. His research work is related to the understanding of the radio propagation channel for UAVs enabled network.

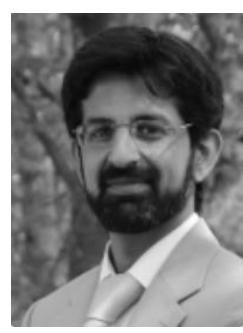

Adnan Ahmad Cheema received the Ph.D. degree in electronics engineering from Durham University, U.K., in 2015. He continued to work in Durham as a Research Associate on a project funded by OFCOM, U.K., dealing with fifth-generation (5G) radio propagation channel measurements and modelling. In 2017, he joined Ulster University, U.K., as a Lecturer in electronics engineering. His research interests include drone communications, radio propagation, and the Internet of Things, particularly for applications in 5G and beyond, disaster management, and e-health.

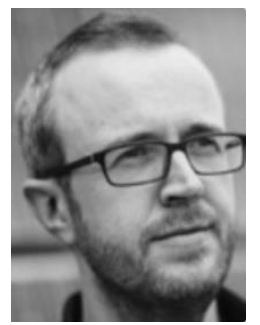

Dewar Finlay is a Professor in Electronic Engineering in the School of Engineering and a member of the Engineering Research Institute. His main area of research interest is in healthcare technology. He has secured funding to support this research from a number of funding bodies that include: EU (H.2020), ESRC, DEL, InvestNI, HEA. He is currently a member of the Board of Directors of Computing in Cardiology Inc. He is a member of the Editorial Board of the Journal of Electro cardiology and has served as conference Chairperson for both the 37th (2010) Computing in Cardiology conference and the 40th (2015) Conference of the International Society for Computerized Electro cardiology. 